Article

\title{
Investigation on the Characteristics of Different Shaped Wave Energy Harvesters based on Semi-analytical Solution
}

\author{
Ming Liu ${ }^{1}$, Hengxu Liu ${ }^{2 *}$, Yuanchao Chai ${ }^{1}$ Liquan Wang1 and Zhiyang Zhang ${ }^{2}$ \\ 1 College of Mechanical and Electrical Engineering, Harbin Engineering University, Harbin 150001, China; \\ lium@hrbeu.edu.cn(M.L.); edisonchai@hrbeu.edu.cn(Y.C.); wangliquan@hrbeu.edu.cn(L.W.) \\ 2 College of Shipbuilding Engineering, Harbin Engineering University, Harbin 150001, China; \\ 15045630022@163.com(Z.Z.) \\ * Correspondence: liuhengxu@hrbeu.edu.cn (H.L.)
}

\begin{abstract}
We mainly describe the influence factors of captured power by huge wave energy harvesters, which the vertical motion of buoy can transform ocean's potential energy into piezoelectric energy power by waves. By means of semi-analytical solutions and theoretical analysis, related environmental coefficients are analyzed firstly and find the most appropriate wave frequency and geometric structure as reference. Secondly, the numerical results also discuss the impact trend of hydrodynamic parameters and geometric volume on motion, voltage and power with qualitative agreement. The simulation data confirm that structure parameters of the modified model could markedly deliver sufficient power to ocean high electrical equipment with long-time stability.
\end{abstract}

Keywords: semi-analytical solutions; hydrodynamic characteristics; piezoelectric cantilever beam; wave energy harvesters; lumped and structural parameters.

\section{Introduction}

As the increasing of resource consumption per capita and ocean possessing the richest natural resources on earth, we begin to explore ocean resources as a promising long research. The wave has quite high kinetic energy and potential energy, which theoretical average power of wave energy can obtain $1.3 \times 10^{7} \mathrm{~kW}$ in coastal areas according to the data of China's coastal ocean observation stations.

At the same time, power supply devices for ocean exploration equipment are continuously complexity. However, the original power supply method is the long-distance transmission voltage by power plant, which cost expensive and heavy losses during power transmission. On another hand, pocket spontaneous electrical equipment cannot meet high-power requirements that lower power input, short endurance, disposable use (once the fault is damaged, the repair is poor.). So to deal with the current thorny problem, we proposed an oversize piezoelectric cantilever beam that converting a wide range of ocean energy into piezoelectric energy power and it can ensure the stable and sufficient voltage to detection equipment without certain restrictions. Meanwhile, improved structure can obviously enhance energy power. Hence, it is a reasonable point and effective way to meet the requirements of offshore power supply system.

The pioneer work can be traced to Taylor et al. [1] who design an energy harvesting eel to capture energy from the vibration and bring up alternate shedding vortex by water currents at the beginning of 21st century. Besides, eel is a relatively inexpensive and easily scalable device, which has capacity to generate from milliwatts to many watts depending on system size and flow velocity of local environment. Moreover, Kui Yao et al. [2] adopt lumped-mass modeling to analyze composite 
piezoelectric cantilever and illustrate general trends associated with changing parameters of the structure and materials. Steven R Anton et al. [3] put forwad a new piezoelectric materials called PZT that feasible PZT provides further development of realizing completely self-powered devices. In addition, A Erturk et al. [4] perform an experiment of basic harmonic excitation at an arbitrary excitation frequency (translation in the transverse direction with small rotation) for steady state voltage. Samuel C. Stanton et al. [5], H. L. Dai et al. [6], N.V. Viet et al. [7] also improve the capacity of Euler-Bernoulli piezoelectric cantilever beam from geometric construction and structure coefficient from experiment and simulation. H. Abdelmoula et al. [8] gives guidelines for designing efficient galloping energy harvesters and characterizes the types of interactions among coupled frequencies of mechanical and electrical types.

Due to the oversize harvester, hydrodynamic calculation needs to be fully analyzed for its stable and efficient of generating capacity. S.Malenica et al. [9] propose a vertical cylinder submitted to regular waves and moderate current in water of finite depth where calculation of mean drift forces, near-field, wave drift damping coefficients, and far-field methods are used and compared with numerical results provided by Grue personal communication. S.A.Mavrakos et al. [10] present linearize exciting wave forces and hydrodynamic parameters of vertical cylinders that are exposed to action of regular surface waves in finite depth water. The previous papers mainly discuss the linear problem and infinite depth in the theoretical analysis and simulation. F. Flocard et al. [11] present from an experimental study on bottom-pivoted pitching point energy absorbers in intermediate water depth from regular and irregular waves, it shows that some parameters can be adjusted in response to captured power in various waves. Robert Valentine Chaplin et al. [12] present another new experimental study of converter where provided the multi-scale test results confirm Seaweaver's strong economic promise a 1/3 version of the design should be built and trialled at sea. Besides, Wanchao Zhang et al. [13] adopt semi-analytical method of decomposing complex axisymmetric boundary into several ring-shaped and stepped surfaces based on the boundary discretization method.

From then on, many researchers have made great contributions to piezoelectric cantilevers beam, which starts with the ideal structure of the harvester to realizable piezoelectric cantilever beam with low voltage. However, the previous experience of piezoelectric cantilever beams only applies to pocket size model that lower output, short endurance, use disposable and little higher energy power under some certain working conditions(such as: expensive materials, high waves, high frequency vibration, and so on). On another hand, piezoelectric cantilever beam is a forward-looking point for its sustainable development, but there are not a majority acknowledged framework and a cost-effective model. Besides, on boundary condition analysis, the environmental factors are not taken into account be fully proved. Furthermore, present structures of piezoelectric cantilever beam are seldom improved to enhance its efficiency of power generation and explicitly analyze the influence curve by buoy's structure and lumped parameters (such as: geometry of buoys, volume of buoy, shape of piezoelectric beam)

In this paper, we will start by applying a spring-mass-damper system to simulate heaving motion of oversize wave energy harvester, which simplify model structure also can ensure the accuracy and rationality of numerical results based on control equation and semi-analytic method. In addition, simulation results can also show added mass, added damping, wave force in a complete logic algorithm chain in which the influence of ocean parameters are discussed and optimum values of environmental parameter are selected as reference. Finally, numerical results graphs can comprehensively show the influence of structural parameters and lumped coefficients on piezoelectric energy power. Furthermore, energy power capacity of piezoelectric cantilever beam 
has been obtained a dramatic improvement because that captured power is large than other wave harvester under same structural parameters which means can transport higher power to exploration equipment for stabilizing the voltage day and night. Besides, this huge piezoelectric cantilever beam can be repaired after repetitious employment.

\section{Materials and Methods}

In this paper, the study object is optimized structural parameters for enhancing captured energy power conversion by the piezoelectric cantilever beam on the ocean platform, which the oversize device is displayed in Figure. 1 (right). The PZT- 5 is used as a piezoelectric medium that is attached to the upper and lower beam surface with equal density and the same volume. At the end of the cantilever beam is a float that can create motion excited by the heaving motion of waves. The Figure 1 shows a conical buoy and it can arbitrary shaped and vertical axisymmetric. Buoys inspired by waves can bend the cantilever beam, resulting in various electric potential difference of parallel piezoelectric layer.

Meanwhile, the outline of the cantilever beam is a trapezoid because that can convert more efficient power than others as same conditions. In order to simplify the calculation process and ensure the effectiveness of results, trapezoidal beam is adjusted into finite equivalent rectangles. Besides, the cantilever beam is assumed that it only has contact with heaving buoy and no external factors attached to buoy. Hence, the motion of harvester under low frequencies can be simplified as lumped-parameter model with a linear spring-mass-damper system and a simple circuit depicted in Figure 1. The water discharge of the buoy is equaled to equivalent concentrated mass of the cantilever beam and the buoy.

Characteristics of motion of buoy are calculated based on semi-analytical method in eigenfunction to evaluate the velocity potentials and calculation of wave force and other parameters. The wetted surface of the buoy and the definition of fluid sub-domain in this study are shown in Figure 1. We define the cylindrical coordinate $(r, \theta, z)$ by its origin located at the center of the buoy and on the mean plane of the free surface. The wetted surface of the buoy is assumed to a floating vertical axisymmetric curved surface. The outer radii and draught of the buoy are denoted as $R$ and $d$ respectively.
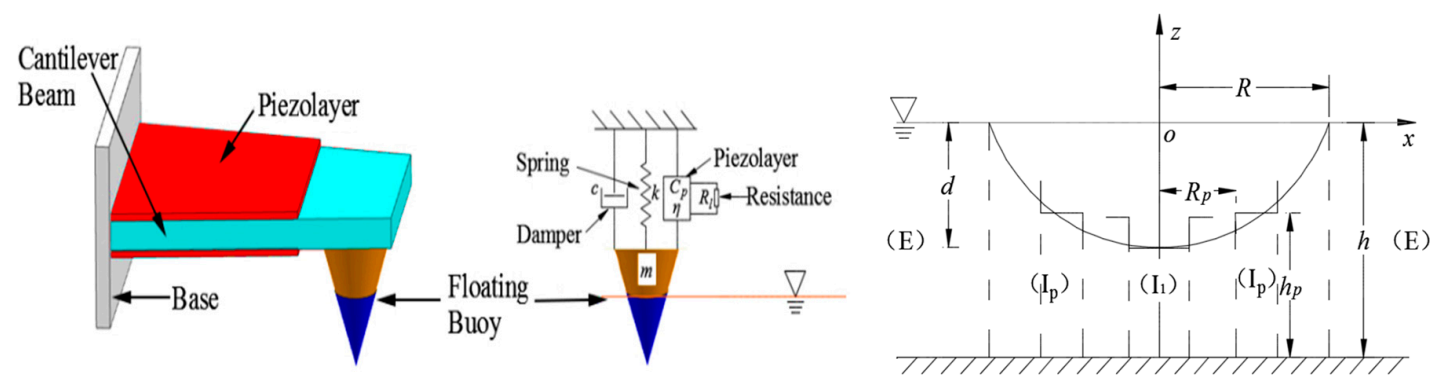

Figure 1. Schematics of a large bimorph piezoelectric cantilever beam and the wetted surface of the buoy and the definition of fluid sub-domain

\subsection{Boundary condition}

According to the linear wave theory in frequency domain analysis, the spatial velocity potential $\Phi$ can be decomposed as undisturbed incident wave velocity potential $\Phi_{0}$, scattered potential $\Phi_{7}$ for fixed buoy and radiation potential $\Phi_{3}$ induced by the buoy heave motion oscillation in otherwise calm water. Then we have,

$$
\Phi=\Phi_{0}+\Phi_{7}+A_{3} \Phi_{3}
$$


where, $A_{3}$ is the displacement amplitude in heave of buoy. In Eq.(11), $\Phi_{0}$, representing the velocity of undisturbed incident wave of amplitude $A$ and frequency $\omega$, which propagates along the positive $\mathrm{x}$-axis direction, can be expressed in cylindrical coordinates as:

$$
\Phi_{0}=-(A g / \omega)\left[\cosh k_{0}(z+h) / \cosh k_{0} h\right] \sum_{\ell=0}^{\infty} \varepsilon_{\ell} i \mathbf{J}_{\ell}\left(k_{0} r\right) \cos \ell \theta
$$

where, $\mathbf{J}_{\ell}(\cdot)$ is the first kind Bessel function of order $\ell(\ell=0,1,2, \ldots)$; the symbol $\varepsilon_{\ell}$ is the Neumann's symbol, defined by $\varepsilon_{0}=1$ and $\varepsilon_{\ell}=2$ for $\ell \geq 1$; wave number $k_{0}$ comes from dispersion relation $k_{0} \tanh k_{0} h=\omega^{2} / g$. According to the potential flow theory, the diffracted and radiated velocity potentials should satisfy,

Laplace's equation:

Free surface condition:

Seabed condition:

Hull boundary condition:

Radiation condition:

$$
\begin{aligned}
& \nabla^{2} \Phi_{3,7}=0 \quad \text { (in fluid domain) } \\
& \omega^{2} \Phi_{3,7}-g \partial_{z} \Phi_{3,7}=0 \quad(z=0) \\
& \partial_{z} \Phi_{3,7}=0 \quad(z=-h) \\
& \left\{\begin{array}{c}
\partial_{n} \Phi_{3}=(-i \omega) n_{3} \\
\partial_{n} \Phi_{7}=-\partial_{n} \Phi_{0}
\end{array} \quad\right. \text { (on the wetted surface of buoy) } \\
& \lim _{r \rightarrow \infty} \sqrt{k_{0} r}\left(\partial_{r} \Phi_{3,7}-i k_{0} \Phi_{3,7}\right)=0
\end{aligned}
$$

where $i$ is the imaginary unit and the symbol $\partial_{n}(\cdot)$ indicates the derivative in the normal vector pointing always outwards the wetted surface of the buoy.

In the same way of incoming wave, diffraction velocity potential $\Phi_{7}$ can be expressed as

$$
\Phi_{7}=-(A g / \omega) \sum_{\ell=0}^{\infty} \varphi_{7, \ell} \cos \ell \theta
$$

The fluid flow caused by the force oscillation for the bodies in otherwise still water is symmetric about both $\theta=0^{\circ}$ - and $\pi / 2$ - panels for heave. Thus the radiation velocity potential

$$
\Phi_{3}=\varphi_{3}
$$

\subsection{Velocity potentials in each sub-domain}

To develop the velocity potentials of diffraction and radiation, the fluid domain due to the floating vertical axisymmetric buoy is divided into $P+1$ sub-domains which named as $\mathrm{E}, \mathrm{I}_{1} \ldots \mathrm{I}_{\mathrm{p}} \ldots \mathrm{I}_{\mathrm{p}}$ shown in Figure 1.

In the sub-domain $\mathrm{E}$, the velocity potentials of diffraction can be expressed in the form of Fourier series by,

$$
\begin{aligned}
\varphi_{7, \ell}^{E}= & \alpha_{7, \ell, 0}^{E}\left[\cosh k_{0}(z+h) / \cosh k_{0} h\right]\left[\mathbf{H}_{\ell}\left(k_{0} r\right) / \mathbf{H}_{\ell}^{\prime}\left(k_{0} R\right)\right] \\
& +\sum_{n=1}^{\infty} \alpha_{7, \ell, n}^{E}\left[\cos k_{n}(z+h) / \cos k_{n} h\right]\left[\mathbf{K}_{\ell}\left(k_{n} r\right) / \mathbf{K}_{\ell}^{\prime}\left(k_{n} R\right)\right]
\end{aligned}
$$

The $\mathbf{H}_{\ell}(\cdot)$ and $\mathbf{K}_{\ell}(\cdot)$ are the first kind Hankel function and the modified second kind Bessel function of order $\ell$ separately. Here and hereafter, a prime denotes taking the differentiation of a function with respect to its argument. The wave number $k_{n}(n=1,2,3 \ldots)$ comes from the dispersion relation $k_{n} \tan k_{n} h=-\omega^{2} / g$. Similar, the velocity potentials of radiation can be expressed

$$
\varphi_{3}^{E}=\alpha_{3,0}^{E}\left[\cosh k_{0}(z+h) / \cosh k_{0} h\right]\left[\mathbf{H}_{0}\left(k_{0} r\right) / \mathbf{H}_{0}^{\prime}\left(k_{0} R\right)\right]+\sum_{n=1}^{\infty} \alpha_{3, n}^{E}\left[\cos k_{n}(z+h) / \cos k_{n} h\right]\left[\mathbf{K}_{0}\left(k_{n} r\right) / \mathbf{K}_{0}^{\prime}\left(k_{n} R\right)\right]
$$

In the sub-domai $I_{1}$, the velocity potentials of diffraction can be expressed

$$
\varphi_{7, \ell}^{\mathrm{I}_{1}}=\alpha_{7, \ell 0}^{\mathrm{I}_{1}}(r / R)^{\ell}+\sum_{n=1}^{\infty} \alpha_{7, \ell, n}^{\mathrm{I}_{1}}\left[\mathbf{I}_{\ell}\left(\lambda_{n, 1} r\right) / \mathbf{I}_{\ell}^{\prime}\left(\lambda_{n, 1} R\right)\right] \cos \lambda_{n, 1}\left(z+h_{1}\right)
$$


The $\mathbf{I}_{\ell}(\cdot)$ are the modified first kind Bessel function of order $\ell$. The $\lambda_{n, 1}$ can be defined as $\lambda_{n, 1}=n \pi / h_{1}$. Similar, the velocity potentials of radiation can be expressed

$$
\varphi_{3}^{\mathrm{I}_{1}}=\alpha_{3,0}^{\mathrm{I}_{1}}(r / R)^{\ell}+\sum_{n=1}^{\infty} \alpha_{3, n}^{\mathrm{I}_{1}}\left[\mathbf{I}_{0}\left(\lambda_{n, 1} r\right) / \mathbf{I}_{0}^{\prime}\left(\lambda_{n, 1} R\right)\right] \cos \lambda_{n, 1}\left(z+h_{1}\right)+\left[2(z+h)^{2}-r^{2}\right] / 4 h_{1}
$$

In the sub-domain $I_{p}(p=2,3 \ldots N)$, the velocity potentials of diffraction can be expressed

$$
\begin{aligned}
\varphi_{7, \ell}^{\mathrm{I}_{p}} & =\alpha_{7, \ell, 0}^{\mathrm{I}_{p}} G_{0}^{\ell}(r)+\sum_{n=1}^{\infty} \alpha_{7, \ell, n}^{\mathrm{I}_{p}}\left[\mathrm{~K}_{I}^{n \ell} \cdot \mathrm{I}_{\ell}\left(\lambda_{n, p} r\right)-\mathrm{I}_{I}^{n \ell} \cdot \mathrm{K}_{\ell}\left(\lambda_{n, p} r\right)\right] \cos \lambda_{n, p}\left(z+h_{p}\right) \\
& +\tilde{\alpha}_{7, \ell, 0}^{I_{p}} \tilde{G}_{0}^{\ell}(r)+\sum_{n=1}^{\infty} \tilde{\alpha}_{7, \ell, n}^{I_{p}}\left[\mathrm{I}_{E}^{n \ell} \cdot \mathrm{K}_{\ell}\left(\lambda_{n, p} r\right)-\mathrm{K}_{E}^{n \ell} \cdot \mathrm{I}_{\ell}\left(\lambda_{n, p} r\right)\right] \cos \lambda_{n, p}\left(z+h_{p}\right)
\end{aligned}
$$

in which

$$
\begin{aligned}
& G_{0}^{\ell}(r)=\left\{\begin{array}{l}
\ln \left(r / R_{p-1}\right) / \ln \left(R_{p} / R_{p-1}\right) \\
{\left[\left(r / R_{p-1}\right)^{l}-\left(R_{p-1} / r\right)^{l}\right] /\left(R_{p} / R_{p-1}\right)^{l}-\left(R_{p-1} / R_{p}\right)^{l}}
\end{array}\right. \\
& \tilde{G}_{0}^{\ell}(r)=\left\{\begin{array}{l}
\ln \left(R_{p} / r\right) / \ln \left(R_{p} / R_{p-1}\right) \\
{\left[\left(R_{p} / r\right)^{l}-\left(r / R_{p}\right)^{l}\right] /\left[\left(R_{p} / R_{p-1}\right)^{l}-\left(R_{p-1} / R_{p}\right)^{l}\right]}
\end{array}\right. \\
& \left\{\mathrm{K}_{I}^{n \ell}, \mathrm{I}_{I}^{n \ell}, \mathrm{K}_{E}^{n \ell}, \mathrm{I}_{E}^{n \ell}\right\}=\frac{\left\{\mathrm{K}_{\ell}\left(\lambda_{n} R_{p-1}\right), \mathrm{I}_{\ell}\left(\lambda_{n} R_{p-1}\right), \mathrm{K}_{\ell}\left(\lambda_{n} R_{p}\right), \mathrm{I}_{\ell}\left(\lambda_{n} R_{p}\right)\right\}}{\mathrm{K}_{\ell}\left(\lambda_{n} R_{p-1}\right) \mathrm{I}_{\ell}\left(\lambda_{n} R_{p}\right)-\mathrm{K}_{\ell}\left(\lambda_{n} R_{p}\right) \mathrm{I}_{\ell}\left(\lambda_{n} R_{p-1}\right)}
\end{aligned}
$$

The $\lambda_{n, p}$ can be defined as $\lambda_{n, p}=n \pi / h_{p}$. Similar, the velocity potentials of radiation can be expressed

$$
\begin{aligned}
\varphi_{3}^{\mathrm{I}_{p}} & =\alpha_{3,0}^{\mathrm{I}_{p}} G_{0}^{0}(r)+\sum_{n=1}^{\infty} \alpha_{3, n}^{\mathrm{I}_{p}}\left[\mathrm{~K}_{I}^{n 0} \cdot \mathrm{I}_{0}\left(\lambda_{n, p} r\right)-\mathrm{I}_{I}^{n 0} \cdot \mathrm{K}_{0}\left(\lambda_{n, p} r\right)\right] \cos \lambda_{n, p}\left(z+h_{p}\right) \\
& +\tilde{\alpha}_{3,0}^{I_{p},} \tilde{G}_{0}^{0}(r)+\sum_{n=1}^{\infty} \tilde{\alpha}_{3, n}^{I_{p}}\left[\mathrm{I}_{E}^{n 0} \cdot \mathrm{K}_{\ell}\left(\lambda_{n, p} r\right)-\mathrm{K}_{E}^{n 0} \cdot \mathrm{I}_{0}\left(\lambda_{n, p} r\right)\right] \cos \lambda_{n, p}\left(z+h_{p}\right)+\frac{2(z+h)^{2}-r^{2}}{4 h_{p} R}
\end{aligned}
$$

In the eigenfunction expansions for the velocity potentials $\varphi$, the sets of unknown Fourier coefficients $\alpha$ are to be determined by taking advantage of orthogonality, in so-called Garrett's method, according to matching of the potentials and its normal derivative on the juncture boundaries (matching surface) shared by subdomains.

\subsection{Matching equations}

On the matching surface between sub-domain $\mathrm{I}_{p}$ and sub-domain $\mathrm{I}_{p+1}$ in which $(1 \leq p \leq P-1)$, the flowing series of functions are expressed as

$$
\begin{gathered}
\int_{-h}^{h_{p}-h} \varphi_{j, l}^{I_{p}}\left(r_{p}, z\right) \mathrm{d} z=\int_{-h}^{h_{p}-h} \varphi_{j, l}^{I_{p+1}}\left(r_{p}, z\right) \mathrm{d} z \\
\int_{-h}^{h_{p}-h} \varphi_{j, l}^{I_{p}}\left(r_{p}, z\right) \cos \lambda_{n_{p}}(z+h) \mathrm{d} z=\int_{-h}^{h_{p}-h} \varphi_{j, l}^{I_{p+1}}\left(r_{p}, z\right) \cos \lambda_{n_{p}}(z+h) \mathrm{d} z \\
\int_{-h}^{h_{p}-h} \partial_{r} \varphi_{j, l}^{I_{p}}\left(r_{p}, z\right) \cosh k_{0}(z+h) \mathrm{d} z=\int_{-h}^{h_{p+1}-h} \partial_{r} \varphi_{j, l}^{I_{p+1}}\left(r_{p}, z\right) \cosh k_{0}(z+h) \mathrm{d} z \\
\int_{-h}^{h_{p}-h} \partial_{r} \varphi_{j, l}^{I_{p}}\left(r_{p}, z\right) \cos k_{n}(z+h) \mathrm{d} z=\int_{-h}^{h_{p+1}-h} \partial_{r} \varphi_{j, l}^{I_{p+1}}\left(r_{p}, z\right) \cos k_{n}(z+h) \mathrm{d} z
\end{gathered}
$$

On the matching surface between sub-domain $\mathrm{I}_{P}$ and sub-domain $\mathrm{E}$, the flowing series of functions are expressed as

$$
\begin{gathered}
\int_{-h}^{h_{p}-h} \varphi_{j, l}^{I_{p}}\left(r_{p}, z\right) \mathrm{d} z=\int_{-h}^{h_{p}-h} \varphi_{j, l}^{E}\left(r_{p}, z\right) \mathrm{d} z \\
\int_{-h}^{h_{p}-h} \varphi_{j, l}^{I_{p}}\left(r_{p}, z\right) \cos \lambda_{n_{p}}(z+h) \mathrm{d} z=\int_{-h}^{h_{p}-h} \varphi_{j, l}^{E}\left(r_{p}, z\right) \cos \lambda_{n_{p}}(z+h) \mathrm{d} z \\
\int_{-h}^{h_{p}-h} \partial_{r} \varphi_{j, l}^{I_{p}}\left(r_{p}, z\right) \cosh k_{0}(z+h) \mathrm{d} z=\int_{-h}^{0} \partial_{r} \varphi_{j, l}^{E}\left(r_{p}, z\right) \cosh k_{0}(z+h) \mathrm{d} z \\
\int_{-h}^{h_{p}-h} \partial_{r} \varphi_{j, l}^{I_{p}}\left(r_{p}, z\right) \cos k_{m}(z+h) \mathrm{d} z=\int_{-h}^{0} \partial_{r} \varphi_{j, l}^{E}\left(r_{p}, z\right) \cos k_{m}(z+h) \mathrm{d} z
\end{gathered}
$$


By solving the above linear equations, the Fourier coefficients can be confirmed.

\subsection{Excitation force and hydrodynamic coefficients}

Considering the heave motion model applied in the wave energy conversion, the wave forcing and radiation damping coefficients in heaven need to be obtained. Thus, by defining $R_{0}=0$, the non-dimensional excitation force in heaven can be calculated and defined by

$$
\mathrm{F}_{d}=2 i \rho g A \pi \cdot \sum_{p=1}^{N} R_{p} \int_{R_{p-1}}^{R_{p}} \varphi_{D, 0}^{I_{p}}\left(r, h_{p}-h\right) r \mathrm{~d} r
$$

The hydrodynamic coefficients containing the added mass $\mu$ and the damping coefficients $\lambda$ can be calculated and defined by

$$
\mu+i \lambda / \omega=2 \pi / \rho R^{3} \cdot \sum_{p=1}^{N} R_{p} \int_{R_{p-1}}^{R_{p}} \varphi_{3,0}^{I_{p}}\left(r, h_{p}-h\right) r d r
$$

\subsection{Governing equations}

According to Newton's law, the dynamical equation of the heaving buoy in time domain can be written as

$$
m \ddot{z}=F_{d}+F_{r}+F_{b}+F_{o}
$$

where $m$ is the equivalent mass of the lumped-parameter model. The symbol $\ddot{z}, \dot{z}$ and $z$ is the vertical accelerated velocity, velocity and displacement of the buoy. The symbol $F_{d}, F_{r}, F_{b}$ and $F_{o}$ are excitation force, wave radiation force, restoring force and force due to the oscillation of the linear spring-mass-damper system. The excitation force will be detailedly discussed in section.3.

The wave radiation force can be written as

$$
F_{r}=-\mu \ddot{z}-\lambda \dot{z}
$$

where $\mu$ is the added mass and $\lambda$ is the damping coefficients, which are functions of the potential flow frequency and will be determined by potential flow theory calculated in section.3.

The restoring force can be written as

$$
F_{b}=-b z
$$

where $b$ is the restoring coefficients, $b=\rho g d$. $\rho$ is the density of the seawater, $g$ is the gravitational acceleration, and $d$ is the mean draught of the buoy.

The oscillation force of the lumped-parameter model is

$$
F_{o}=-c \dot{z}-k z+\eta v
$$

where $c, k$ and $\eta$ are the equivalent damping coefficient, the equivalent spring coefficient, and the electromechanical coupling of the piezoelectric cantilever beam, respectively. $v$ is the voltage across the load resistance.

Then, Eq.(1) can be obtained as

$$
(m+\mu) \ddot{z}+(c+\lambda) \dot{z}+(k+b) z-\eta v=F_{d}
$$

According to Kirchhoff's law, the electrical equation of the system in time domain can be written as

$$
\begin{aligned}
& C_{p} \dot{v}+\frac{1}{R_{l}} v+\eta \dot{z}=0 \\
& C_{p} \dot{v}+\frac{1}{R_{l}} v+\eta \dot{z}=0
\end{aligned}
$$

Where $R_{l}$ is the resistance, $C_{p}$ is the capacitance of the piezoelectric material. Since the frequencies of wave are relative lower, $C_{p}$ and $\eta$ can be obtained from the fundamental vibration mode of the piezoelectric cantilever beam. 
According to series piezoelectric layer, electromechanical coupling of harvesting can be written as:

$$
\eta=\left.\overline{e_{31}} b h_{\overline{p c}} \frac{d \phi(x)}{d(x)}\right|_{x=L}
$$

where $\overline{e_{31}}$ is piezoelectric constant, $h_{p c}$ is distance between the center of the piezoelectric layer and the neutral axis.

According to the normalized quality eigenfunction of vibration cantilever beam, it can be obtained as:

$$
\phi(\mathrm{x})=A_{1} \cos \left(\frac{\lambda}{l} \mathrm{x}\right)+A_{2} \cosh \left(\frac{\lambda}{l} \mathrm{x}\right)+A_{3} \sin \left(\frac{\lambda}{l} \mathrm{x}\right)+A_{4} \sinh \left(\frac{\lambda}{l} \mathrm{x}\right)
$$

where $A_{1}, A_{2}, A_{3}, A_{4}$ is corresponding amplitude constant. $\lambda$ is dimensionless frequency of the model (eigenvalue) and $\lambda=l \sqrt{\frac{\rho A \omega^{2}}{E I}}$

Electromechanical coupling of harvesting can be written as:

$$
C_{p}=\frac{\overline{\varepsilon_{33}} b L}{2 h_{\bar{p}}}
$$

where $\overline{\varepsilon_{33}}$ is permittivity of constant force.

\subsection{Average power}

In the frequency domain analysis, in one period, the average power due to piezoelectric effect can be written as

$$
P=\frac{1}{T} \int_{0}^{T} \frac{v^{2}}{R_{l}} d t
$$

using $v=\Re\left\{V e^{j \omega t}\right\}$, so

$$
P=\frac{1}{T R} \int_{l}^{T} \Re\left\{V e^{j \omega t}\right\} \Re\left\{V e^{j \omega t}\right\} d t
$$

using formula

$$
\mathfrak{R}\left\{A e^{j \omega t}\right\} \mathfrak{R}\left\{B e^{j \omega t}\right\}=\frac{1}{2} \mathfrak{R}\left\{A B^{*}+A B e^{2 j \omega t}\right\}
$$

where " is the symbol of a conjugate complex number. Then we have,

$$
P=\frac{1}{2 T R_{l}} \int_{0}^{T} \Re\left\{v v^{*}+v v e^{2 j \omega t}\right\} d t=\frac{v v^{*}}{2 R_{l}}
$$

\section{Numerical results and discussion}

Firstly, environmental parameters be comprehensively considered because that it is an important boundary reference in the calculation of converted ocean energy into piezoelectric energy power, which oversize harvester could capture much more energy as coupled with ocean parameters. First of all, the selected incident wave average frequency range from $4.0 \mathrm{rad} / \mathrm{s}$ to $6.0 \mathrm{rad} / \mathrm{s}$ in this paper. And according to theory of linear wave diffraction can conclude $\lambda / D \leq 5.0$ to ensure the accuracy of the calculation of buoy-motion where $\lambda$ means incident wave frequency and $D$ express ocean depth, besides, the linear diffraction wave theory can analyze corresponding wave force and additional mass of buoy under changing wave frequency. Second, in order to prevent the breaking of diffraction wave in the wave energy conversion process, the breaking wave theory is introduced $A / \lambda \leq 0.14$ where A present wave amplitude, $\lambda=2 \pi / k$ and $k$ is the wave number. Furthermore, 
dispersion equation is carried out to simplify related parameters which show $k \tanh =\omega^{2} / g$. Finally we derive related formula and obtain corresponding data as showed in Table 1.

Table 1. Partly wave characteristic data.

\begin{tabular}{|c|c|c|c|c|c|c|}
\hline $\begin{array}{l}\text { Amplitude } \\
\qquad \mathbf{A}(\mathbf{m})\end{array}$ & $\begin{array}{l}\text { Characteristic } \\
\text { length } 2 R(m)\end{array}$ & $\begin{array}{l}\text { Frequency } \\
\omega(\mathrm{rad} / \mathrm{s})\end{array}$ & $\begin{array}{c}\text { Wavenumber } \\
\qquad\left(\mathbf{m}^{-1}\right)\end{array}$ & $\begin{array}{c}\text { Wave length } \\
\lambda \quad(\mathbf{m})\end{array}$ & $\lambda / \mathbf{2} \mathbf{R}$ & $\mathbf{A} / \lambda$ \\
\hline 0.50 & 1.00 & 3.00 & 0.92 & 6.85 & 6.85 & 0.073044 \\
\hline 0.50 & 1.00 & 3.50 & 1.25 & 5.03 & 5.03 & 0.099421 \\
\hline 0.50 & 1.00 & 4.00 & 1.63 & 3.85 & 3.85 & 0.129856 \\
\hline 0.50 & 1.00 & 4.50 & 2.06 & 3.04 & 3.04 & 0.164349 \\
\hline 0.50 & 1.00 & 5.00 & 2.55 & 2.46 & 2.46 & 0.202900 \\
\hline 0.50 & 1.00 & 5.50 & 3.67 & 1.71 & 1.71 & 0.292176 \\
\hline
\end{tabular}

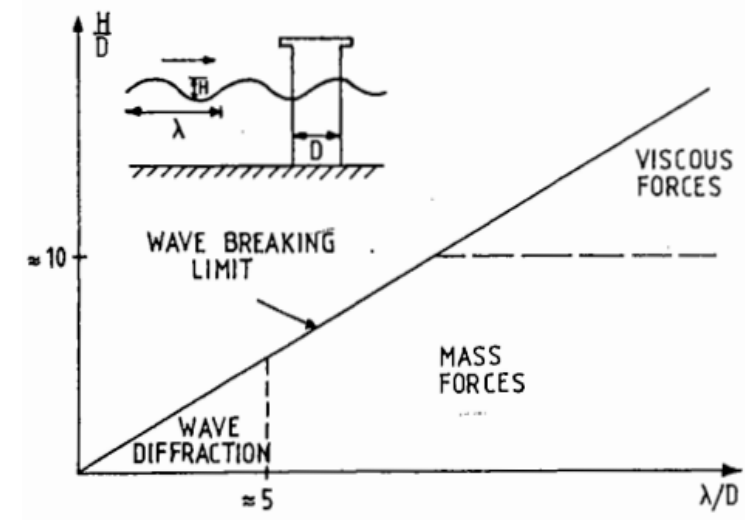

Figure 2. Relative importance of mass, drag and diffraction forces on marine structures.

In order to judge when viscous effects or different types of potential flow are important, it is useful to refer to a simple picture like Figure 2 (From Sea Loads on Ships and Offshore Structures by O.M.Faltinsen). Hence, the incident wave average frequency is selected as $4.0 \mathrm{rad} / \mathrm{s}$ which satisfy linear wave diffraction theory and also meet breaking wave theory under $A=0.5 \mathrm{~m}$ then the following numerical results are dissected to the influence of structural parameters on piezoelectric energy power with certain reference. On the another hand, main objective is to focus on the optimal value of geometrical volume among cone, cylinder and hemisphere for the vertical motion buoy in semi-analytical method and discuss the influence of lumped parameters on the motion, voltage and power. Based on the governing equation and boundary formula, we can get the variable coefficients including basic geometrical volume, add mass $\mu$, damping coefficient $\lambda$, wave diffraction force $F_{d}$, spring stiffness $k$, damping coefficient ${ }^{c}$. For avoiding error of some parameter's factor to analyze numerical results of this oversize harvester, the control variable method is used to ensure the accuracy of the results that set volume $V=0.6 \mathrm{~m}^{3}$, spring coefficients $k=14576 \mathrm{~N} / \mathrm{m}$, damping coefficients $C=525 \mathrm{~N} \cdot \mathrm{m} / \mathrm{s}$, resistance $R_{l}=550 \mathrm{k} \Omega$, capacity $C_{p}=3.9 \times 10^{-7} \mathrm{~F} / \mathrm{m}$, electromechanical coupling $\eta=4.2 \times 10^{-3} \mathrm{C} / \mathrm{N}$ as basic reference. 


\subsection{The effects of geometrical volume $V$}

To discover whether $V$ correlates with collected piezoelectric energy power and following will discuss the influnce of $V$ on related power generation indicators. In addition, we set three groups of different model to analyze and guarantee the accuracy of generating capacity with $\omega=4.0 \mathrm{rad} / \mathrm{s}$.
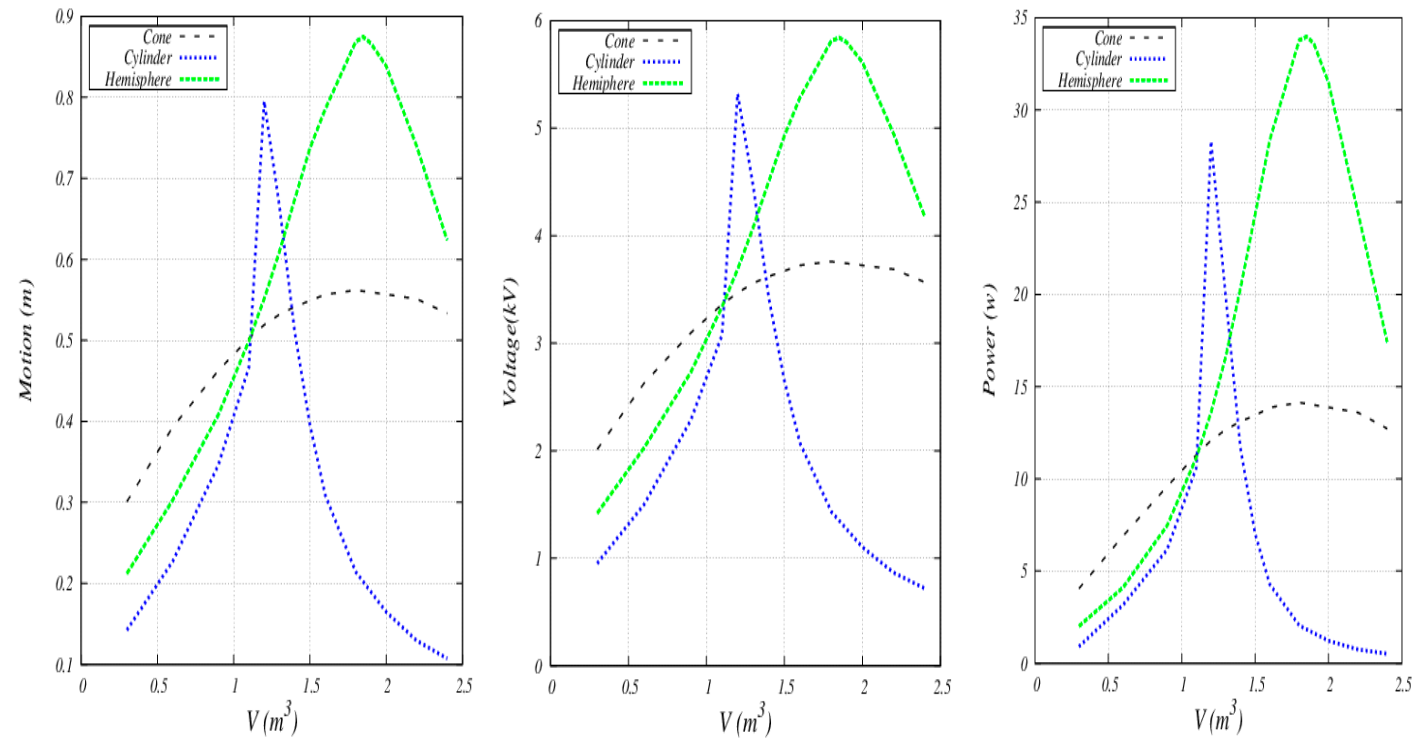

Figure 3. Piezoelectric energy power with increasing $V$; left: Motion; middle: Voltage; right: Power for $\omega=4.0 \mathrm{rad} / \mathrm{s}$.

As Figure 3 shows the comprehensive influence of increasing volume of harvester on captured motion, genreraed voltage and power with constant $\omega$, which demonstrates that motion produced by wave is non-linear. For example, the motion of conical buoy rises gently then goes down with the range of volume changing from $V=0.0 \mathrm{~m}^{3}$ to $V=2.5 \mathrm{~m}^{3}$ and gets its maximum value at Montion $=0.56 \mathrm{~m}$ by $V=1.8 \mathrm{~m}^{3}$. In contrast, cylindrical motions go sharply increasing and decreasing with enlarging volume. For instance, produced motion is less than $0.3 \mathrm{~m}$ when $V$ is less than $V=0.5 \mathrm{~m}^{3}$, but obviously its maximum value of motion produced is $M=0.79 \mathrm{~m}$ at $V=1.7 \mathrm{~m}^{3}$. However, the hemispherical motion is slowly than that of in the changing volume compared to the cylinder and its maximum value is $0.86 \mathrm{~m}$ which is greater than other two basic models. From above graphs, we can conclude a similar pattern of voltage and piezoelectric energy with motion, which its maximum index is $5.8 \mathrm{kV}$ and $35.0 \mathrm{~W}$ by hemisphere, respectively. There are two reasons for the larger $V$ generating more powers. One is that its hydrostatic recovery stress of large $V$ is bigger than smaller ones. The second point is that its resonance volume is different from basic structure with $\omega=4.0 \mathrm{rad} / \mathrm{s}$. As the result, we should choose a hemisphere with $V=1.8 \mathrm{~m}^{3}$ when the stress analysis of a cantilever beam is within a reasonable range.

\subsection{The influence of spring stiffness $k$}

Spring stiffness is a structural parameter and plays a significant role as showed in the work principle diagram (Figure 1), which connects with harvester in simplified models. The subsection will discuss the effects of $k$ on captured indicators in hydrodynamic analysis and calculation from $k=10 \mathrm{kN} / \mathrm{m}$ to $k=70 \mathrm{kN} / \mathrm{m}$. 

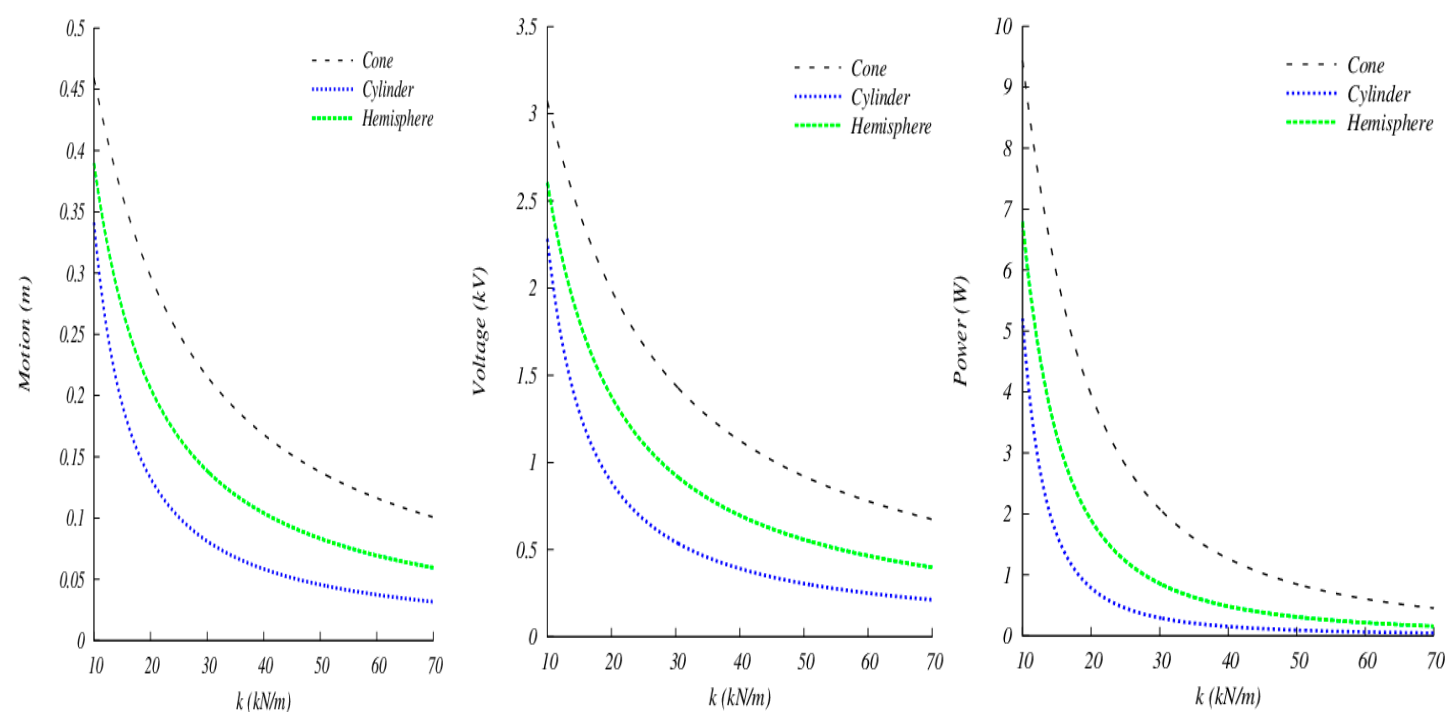

Figure 4. Piezoelectric Energy Power with $k$; left: Motion; middle: Voltage; right: Power for $\omega=4.0 \mathrm{rad} / \mathrm{s} V=0.6 \mathrm{~m}^{3}$.

By looking at the above three graphs in Figure 4, in which reasonably illustrate the influence of growing $k$ on captured motion, voltage and power. Here we can obviously discover the value of motion decline with the increasing $k$ under $V=0.6 \mathrm{~m}^{3}$. For instance, value of conical captured motion drops from $0.46 \mathrm{~m}$ to $0.11 \mathrm{~m}$ in a concave curve while the motions of cylinder and hemispherical are similar than cone under same conditions. As mentioned before, the converted voltage and power highly depend on the motion of harvester, therefore, it can find the graphs of voltage and power also show downward trend for given an increasing $k$ in the upper graphs, which the conical produced voltage and power decline from $3.1 \mathrm{Kv}$ to $0.8 \mathrm{Kv}, 9.2 \mathrm{~W}$ to $0.9 \mathrm{~W}$, respectively. The premier reason is that the greater the stiffness of spring, the worse the deformation ability, which leads to less the motion of harvester in the same $\omega$. In brief, small spring stiffness cannot guarantee the security of model connection when wave frequency is high, the larger spring stiffness can obtain the stability of the conversation but the piezoelectric power will obviously decrease, hence, basing on the previous calculation formula can conclude that $k=14576 \mathrm{~N} / \mathrm{m}$ is the optimal value.

\subsection{The influence of damping coefficients $c$}

Based on above work diagram find the damping coefficient is another significant parameters on the device, which wave contact with harvester that will exert wave damping on the surface of buoy. The following will discuss the influence of damping coefficient on oversize modified piezoelectric cantilever beam under $\omega=4.0 \mathrm{rad} / \mathrm{s}, \quad V=0.6 \mathrm{~m}^{3}$. 

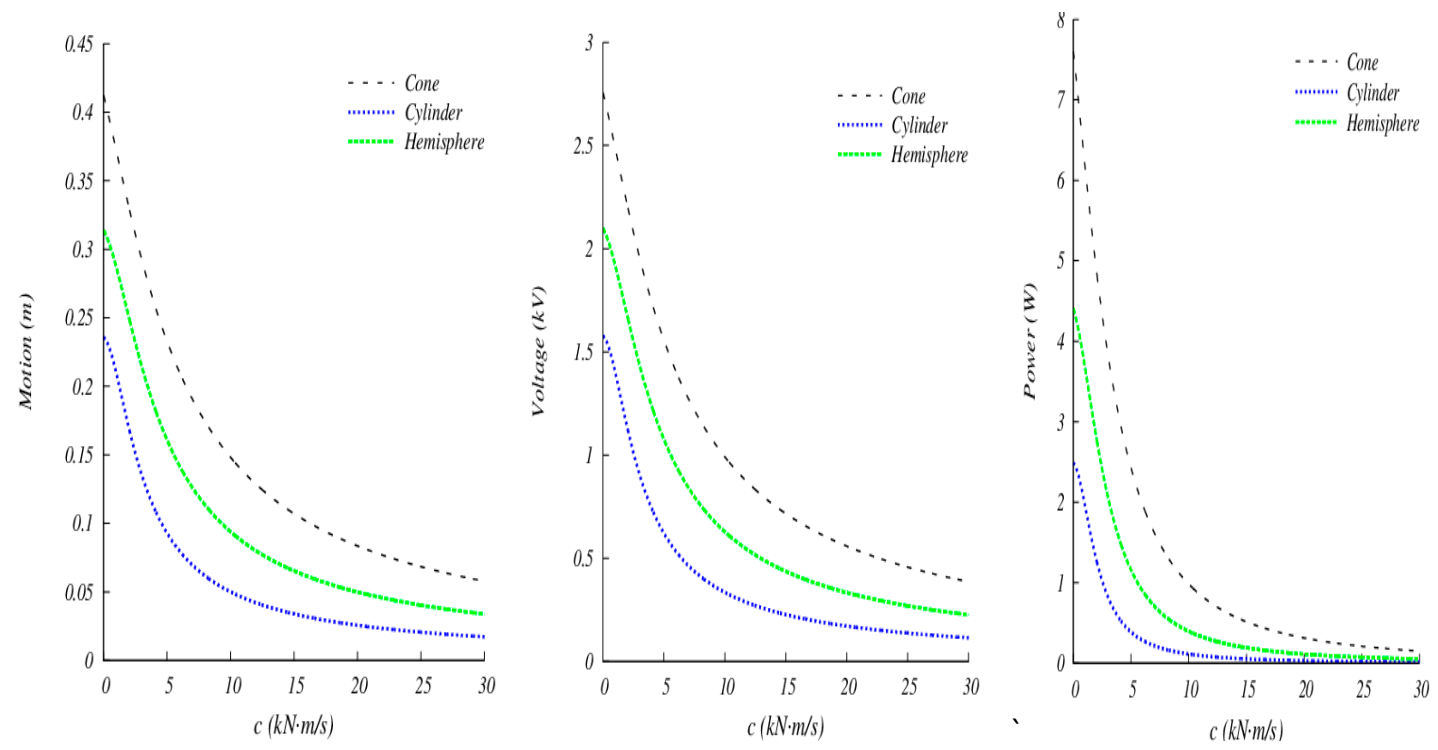

Figure 5. Piezoelectric Energy Power with $c$; left: Motion; middle: Voltage; right: Power for $\omega=4.0 \mathrm{rad} / \mathrm{s}, V=0.6 \mathrm{~m}^{3}$.

In this part, radiation damping is calculated conveniently and feasibility by semi-analytical method of the potential flow theory and linear wave theory. It can be seen the captured motion, produced voltage and power apparently decline with the increasing $c$ among cone, hemisphere and cylinder in the upper graphs Fig.8. For example, value of conical motion goes straight down from $0.41 \mathrm{~m}$ to $0.07 \mathrm{~m}$ therefor its motion drops slowly after $c=20.0 \mathrm{kN} \cdot \mathrm{m} / \mathrm{s}$. And others basic models have similar trends just smaller in numerical results, which the cylindrical energy power decline from $2.7 \mathrm{~W}$ to $0.5 \mathrm{~W}$. The conical buoy is outperformed than others that has been demonstrated in the upper section. In such case, the reason for this is that flowing wave make buoy's surface too much additional mass for increasing ${ }^{c}$, which leads to less motion under the same condition. Furthermore, harvester converts wave energy through the motion of buoy and more motion captured, the more voltage and power produced. As the results, the small damping coefficient makes the piezoelectric beam at high frequency while excessive damping coefficient will affect the piezoelectric energy power, such as over-damping only generate less than $0.1 \mathrm{~W}$ after $c=20.0 \mathrm{kN} \cdot \mathrm{m} / \mathrm{s}$, hence, basing on the parameters and geometric parameters of piezoelectric cantilever beam, reasonable small value of $c$ need accurately to be calculated.

\section{Conclusions and expectation}

In this paper, we will mainly provide a power supply device for spontaneous electricity at sea, which is an oversize piezoelectric cantilever beam by wave loading on its surface without restrictions. First, the boundary equilibrium equation is connected to model to verify the accuracy and facility of numerical values. Furthermore, the optimal frequency is selected by a semi-analytical method in the same ideal conditions and modified model is carried out to enhance energy conversion of from ocean waves among the common basic model by selecting the frequency. Besides, the influence of structural coefficient and lumped parameters on power generation capacity of piezoelectric cantilever beam is described in detail as numerical analysis.

As the results, hemisphere buoy shows an excellent performance than others, which could generates $33.8 \mathrm{~W}$ with a series of certain parameters, respectively. Meanwhile, the enlarged buoy also show an explicit enhance on piezoelectric energy power at the same conditions. And the lumped parameters show a great role in the efficiency of piezoelectric energy power output as well. 
Acknowledgements: This paper is financially supported by the National Natural Science Foundation of China (No.51509048) and National key laboratory research fund for Marine engineering in 2017 (No.1703).

Author Contributions: All the authors have worked hard to discuss the harvester, optimize the calculations and analyze the numerical results. Liu Ming propose ideal model and deduce the formula. Liu Hengxu mainly analyzes environment effects and puts forward reasonable parameters. Chai Yuanchao performs simulation preparation and processing numerical results. Wang Liquan and Zhang Zhiyang provide advices for this paper. We wrote the manuscript together.

Conflicts of Interest: The authors declare no conflict of interest.

\section{References}

1. Taylor, G. W.;Burns, J. R.; Kammann, S. A.; Powers, W. B.; Welsh, T. R. The Energy Harvesting Eel: a small subsurface ocean/river power generator. IEEE Journal of Oceanic Engineering 2001, 26, 539-547.

2. Yao, K.; Uchino, K. Analysis on a composite cantilever beam coupling a piezoelectric bimorph to an elastic blade. Sensors \& Actuators A Physical 2001, 89, 215-221.

3. Anton, S. R.; Sodano, H. A. A review of power harvesting using piezoelectric materials(2003-2006). Smart Materials and Structures 2007, 16, R1-R21.

4. Longini, I. M.; Jr, Ackerman, E.; Kemper, J. An experimentally validated bimorph cantilever model for piezoelectric energy harvesting from base excitations. Smart Materials $\mathcal{E}$ Structures 2009, 18, 25009-25018.

5. Stanton, S. C.; McGehee, C. C.; Mann, B. P. Nonlinear dynamics for broadband energy harvesting: Investigation of a bistable piezoelectric inertial generator. Physica D: Nonlinear Phenomena 2010, 239, 640-653.

6. Dai, H. L.; Abdelkefi, A.; Wang, L. Piezoelectric energy harvesting from concurrent vortex-induced vibrations and base excitations. Nonlinear Dynamics 2014, 77, 967-981.

7. Viet, N.V.; Xie, X.D.; Liew, K. M., Banthia, N.; Wang, Q. Energy harvesting from ocean waves by a floating energy harvester. Energy 2016, 112, 1219-1226.

8. Abdelmoula, H.; Abdelkefi, A. Investigations on the presence of electrical frequency on the characteristics of energy harvesters under base and galloping excitations. Nonlinear Dynamics 2017, 89, 2461-2479.

9. S.Malenica; Clark, P. J.; Molin, B. Wave and current forces on a vertical cylinder free to surge and sway. Applied Ocean Research 1995, 17, 79-90.

10. S Mavrakos. Hydrodynamic characteristics of two concentric surface - Piercing floating circular cylinders. Applied Maritimw Transportation and exploitation Ocean and Coastal Resources 1995, ISBN 0415390362

11. Flocard, F.; Finnigan, T. D. Experimental investigation of power capture from pitching point absorbers. European Wave and Tidal Energy Conference 2009.

12. Chaplin, R. V. Seaweaver:a new surge-resonant wave energy converter. Renewable Energy 2013 ,57, 662-670.

13. ZHANG, W.C.; LIU, H.X.; ZHANG, L.; ZHANG, X.W. Hydrodynamic analysis and shape optimization for vertical axisymmetric wave energy converters. China Ocean Engineering 2016, 30, 954-966.

14. CHEN X.B; LIU H.X; DUAN W.Y. Semi-analytical solutions to wave diffraction of cylindrical structures with a moonpool with a restricted entrance. Journal of Engineering Mathematics 2015, 90, 51-66.

15. JBAILY A.; YEUNG R.W. Piezoelectric devices for ocean energy: a brief survey. Journal of Ocean Engineering and Marine Energy 2015, 1, 101-18.

16. Xie, X.D.; Wang, Q.; Wu, N. Energy harvesting from transverse ocean waves by a piezoelectric plate. International Journal of Engineering Science 2014, 81, 41-48.

17. XIE, X. D.; CARPINTERI, A.; WANG, Q. A theoretical model for a piezoelectric energy harvester with a tapered shape. Engineering Structures 2017, 144, 19-25. 
13 of 13

18. Mukhanov, A.; Abdigaliyev, A.; Jangeldinov, B.; Zhussip, M.; Zhapparov, R.; Ruderman, A.; Adair, D. Development of a Design Tool for Optimization of Voltage Generation from a Bimorph Piezoelectric Cantilever Beam. Materials Today: Proceedings 2017, 4, 4477-4490.

19. Firoozy, P.; Khadem, S. E. and Pourkiaee; S. M. Broadband energy harvesting using nonlinear vibrations of a magnetopiezoelastic cantilever beam. International Journal of Engineering Science 2017, 111, 113-133.

20. Jin, L.; Gao, S.; Zhou, X.; Zhang, G. The effect of different shapes of cantilever beam in piezoelectric energy harvesters on their electrical output. Microsystem Technologies 2017, 23, 4805-4814,

21. Stansby, P.; Carpintero, M.E.; Stallard T.; Maggi, A. Three-float broad-band resonant line absorber with surge for wave energy conversion. Renewable Energy 2015, 78, 132-140.

22. Mccabe, A.P.; Aggidis, G.A.; Widden, M.B. Optimizing the shape of a surge-and-pitch wave energy collector using a genetic algorithm. Renewable Energy 2010, 35, 2767-2775. 